\title{
CASCADE CONTROL OF UNSTABLE SYSTEMS WITH APPLICATION TO STABILIZATION OF SLUG FLOW
}

\author{
Espen Storkaas* Sigurd Skogestad ${ }^{*, 1}$ \\ * Department of Chemical Engineering, \\ Norwegian University of Science and Technology, \\ Trondheim, Norway
}

\begin{abstract}
The topic of this paper is the effect of stabilizing control on the remaining control problem. In many cases there is no effect. However, stabilization requires the active use of inputs, and the underlying unstable pole will appear as an undesirable unstable zero if we are concerned with input performance. The implications of this are clearly demonstrated on the application to stabilization of severe slugging in two-phase pipeline-riser systems. We find that a controllability analysis gives important information for measurement selection and performance limitations.
\end{abstract}

Keywords: Cascade control, unstable, instability, controllability, performance limitations

\section{INTRODUCTION}

When unstable (RHP) poles are present in a system, these need to be stabilized, preferably by low-level (secondary) control. Skogestad et al. (2002) discuss the control limitations imposed by a RHP pole with focus on the stabilizing control loop. They show that a RHP pole imposes a lower limit on the $\mathcal{H}_{2}$ - and $\mathcal{H}_{\infty}$ - norm of the transfer function KS from outputs to inputs, and that the unstable pole manifests itself as a RHP-zero in KS, limiting input movement.

The stabilizing loop needed when a RHP pole is present is usually part of a larger control system. The topic of this paper is the effect unstable poles and its manifestations have on the higher levels in the system. We will briefly discuss the open loop behavior for a process with stabilized RHP poles, and study in more detail a cascade control system

\footnotetext{
1 Author to whom correspondence should be addressed. email: skoge@chemeng.ntnu.no
}

where the inner loop stabilizes the unstable poles of the system.

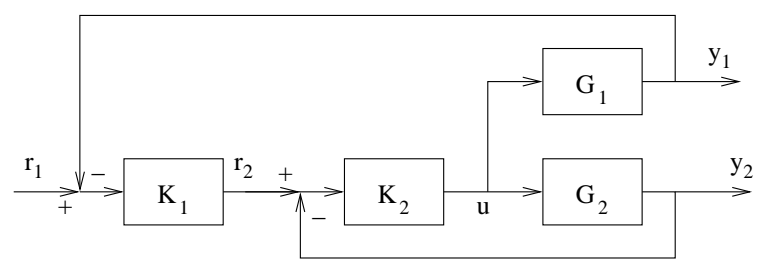

Fig. 1. Block diagram for a cascade control system, 1 - primary (outer) loop, 2 - secondary (inner) loop

We will use the cascade configuration given in Figure 1. $G_{1}(s)$ and $G_{2}(s)$ are the transfer functions from the input $u(s)$ to the primary output $y_{1}(s)$ and the secondary output $y_{2}(s)$, respectively. We will omit the argument $(s)$ in the following to simplify the notation. We assume that $G_{2}$ has at least one unstable pole. $K_{2}$ is the controller for the inner loop, and $K_{1}$ is the controller for the outer loop. The output $y_{1}$ from $K_{1}$ is the reference signal to $K_{2}$. Both controllers are assumed stable. 
Two different cases will be studied, one where the unstable modes are detectable $\left(G_{1}\right.$ contains the same unstable poles as $G_{2}$ ), and one where the unstable modes are not detectable in $y_{1}\left(G_{1}\right.$ is open-loop stable). Our main example will be the stabilization of gravity induced slug flow in multiphase pipeline-riser systems. This system is excellent for demonstrating the importance for controllability analysis and the limitations imposed by non-minimum-phase systems, as it contains both RHP poles and RHP zeros as well as other control limitations depending on the choice of controlled outputs. It also contains both the alternatives for $G_{1}$ described above.

\section{LIMITATIONS IMPOSED BY UNSTABLE POLES AND ZEROS}

Consider a plant with state space matrices A, $\mathrm{B}, \mathrm{C}$ and $\mathrm{D}$, and transfer function $G(s)=$ $C(s I-A)^{-1} B+D$. The poles of the plant are the eigenvalues of $\mathrm{A}$, and the plant is unstable if the poles are in the RHP plane. By the right-half plane we mean the closed right half of the complex plane, including the imaginary axis ( $\mathrm{j} \omega$-axis).

Unstable poles need feedback for stabilization, the presence of RHP poles places a lower band on the bandwidth of the feedback system. For a real pole p, Skogestad and Postlethwaite (1996) gives the lower limit $\omega_{c}>2 p$, while for an imaginary pole the limit is $\omega_{c}>1.15|p|$.

Zeros usually arise when competing effect, internal to the system, results in a zero output from a transfer function for non-zero inputs. We will later show that RHP zeros also can arise from stabilization of RHP poles. For a SISO system, the zeros $z_{i}$ are the solutions to $G\left(z_{i}\right)=0$. RHP zeros give rise to inverse response behavior, as the output from a stable plant with $n_{z}$ RHP zeros will cross its original value $n_{z}$ times as response to a step change in its input (Holt and Moriari, 1985)(Rosenbrock, 1970).

It is also well known form classical root-locus analysis that as the feedback gain increases towards infinity, the closed loop poles moves towards the open-loop zeros. This implies high gain instability and bandwidth limitations. Skogestad and Postlethwaite (1996) derives the following upper bandwidth limitations for systems with RHP zeros.

Real zero:

$$
\omega_{B} \approx \omega_{c}<\frac{z}{2}
$$

Complex zeros

$$
\omega_{B} \approx \omega_{c}< \begin{cases}|z| / 4 & \operatorname{Re}(z) \gg \operatorname{Im}(z) \\ |z| / 2.8 & \operatorname{Re}(z)=\operatorname{Im}(z) \\ |z| & \operatorname{Re}(z) \ll \operatorname{Im}(z)\end{cases}
$$

When both RHP poles and zeros are present in a system, the presence of the above mentioned upper and lower bandwidth limitations may render stabilization impossible. To see this consider the effect of a pair of unstable complex poles with dominant imaginary part $(\operatorname{Re}(p) \ll \operatorname{Im}(p))$ and magnitude $|p|$ and a single real RHP zero z. For the bandwidth limitations to be met, we must approximately require

$$
z>2.3|p|
$$

in order to get acceptable performance and robustness.

\section{FUNDAMENTAL ALGEBRAIC LIMITATIONS}

With the inner stabilizing loop $\left(K_{2}\right)$ closed, the transfer function for the remaining control problem becomes:

$$
\begin{gathered}
G=G_{1} S_{2} K_{2} \\
S_{2}=\left(I+K_{2} G_{2}\right)^{-1}
\end{gathered}
$$

where $G$ is the transfer function from $r_{2}$ to $y_{1}$. To ensure internal stability, unstable poles in $G_{2}$ cannot be cancelled by $K_{2}$. Then, by (5), $S_{2}$ must have RHP-zeros in the same location as the unstable poles in $G_{2}$ if internal stability is to be achieved. If the unstable modes are observable in $y_{1}\left(G_{1}\right.$ contains the same unstable poles as $\left.G_{2}\right)$, the RHP-zeros in $S_{2}$ will be cancelled in $G$. In this case, any bandwidth limitations due to RHP-zeros must come from $G_{1}$.

If the unstable modes in $G_{2}$ are not present in $G_{1}$, as with input reset in the outer loop $y_{1}=u$ $\left(G_{1}=I\right), G$ will have RHP zeros at the location of the RHP poles of $G_{2}$. These RHP zeros will limit the bandwidth of the higher level control system, i.e. for the outer loop in the cascade system in Figure 1.

\section{IMPLICATIONS OF STABILIZED RHP POLES}

If the RHP-zeros originating from the unstable poles of $G_{2}$ are not cancelled by $G_{1}, G$ will have RHP zeros resulting in an inverse response through the process. The paradox for control is that the slower the instabilities in $G_{2}$ (easy stabilization), the slower the inverse response through 
$G$ and the lower the allowed bandwidth (slow control) in the higher levels in the control hierarchy. In other words, the harder job the inner loop controller has, the better control can be expected from the outer loop.

Consider a process $G_{2}$ with a real unstable pole located at $\mathrm{p}$. The sensitivity function $S_{2}$ for the stabilized system will have a RHP zero at p. The bandwidth limitations for the outer loop in the cascade will in our case be $\omega_{B} \approx p / 2$ ( see (1)). This confirms that for slow instabilities (p small) the bandwidth limitations are more severe than for fast instabilities ( $p$ large).

A practical example can be found by trying to keep your balance on a bicycle while staying at the same place. This is obviously an unstable system, and you will have to use your body as a controller to hold your balance. You will find it easier to keep your balance the more the bike is tilted over to the side, as the instability gets slower. However, since you use your body to stabilize the unstable bike, you will find that there is an inverse response in trying to tilt the bike.

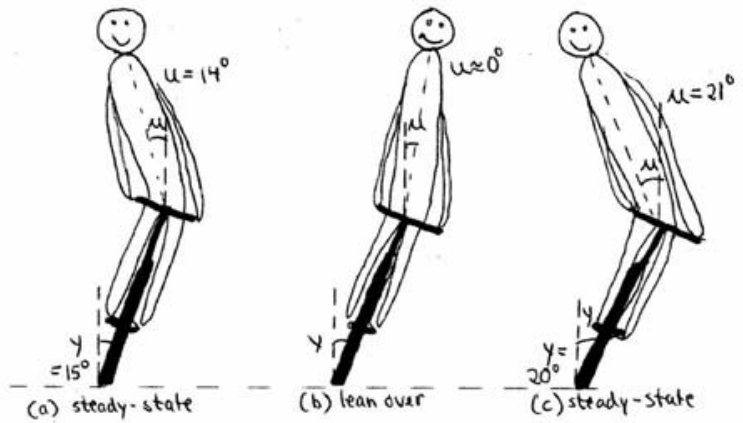

Fig. 2. Inverse response for a bicycle caused by an underlying instability

Consider Figure 2 where the aim is to tilt the bike from an initial angle $y=15^{\circ}$ (Fig. 2a) using your body $(\mathrm{u})$ to an angle $y=20^{\circ}$ (Fig. 2c). Because of the inverse response, you first have to tilt your body in the direction of the tilt to start the movement (Fig. 2b). Eventually, you will have to move your body back to restore balance. This inverse response will be slower the greater the angle y, changing the angle while keeping balanced gets progressively slower as the tilting angle is increased.

\section{CASE STUDY}

Multiphase flow in pipelines differs from regular single phase flow in that a wide variety of flow patterns, also called flow regimes, can develop, dependent on the flow rates, fluid properties and pipeline geometry. Gravity-induced slug flow occurs as a result of a lowpoint connected to an inclining section of the pipe. The pressure drop in

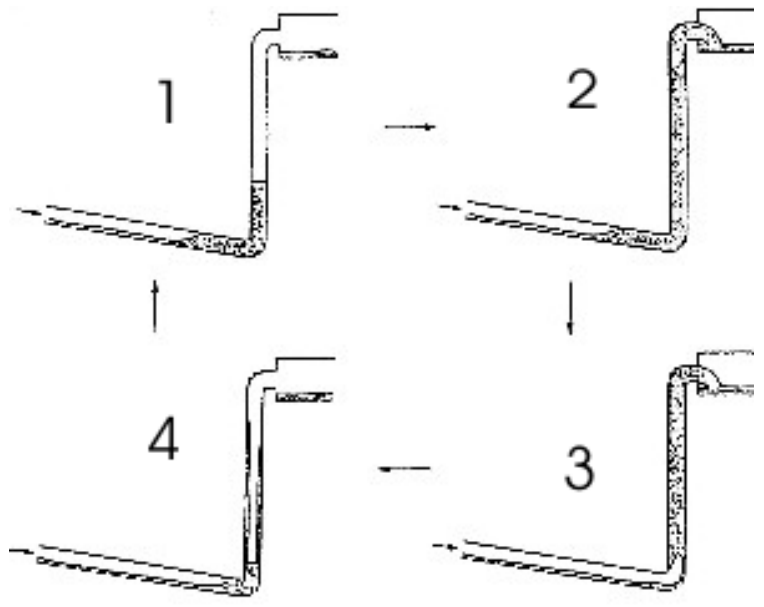

Fig. 3. Illustration of the cyclic behavior (slug flow) in pipeline-riser systems

the pipeline and the interphase friction between the phases are in these cases not sufficient to transport the liquid uphill in a stationary fashion. The liquid will accumulate in the lowpoint, and a liquid slug will form.

The liquid slug that forms will block the flow of gas in the pipe, and grow until enough upstream pressure has developed to overcome the weight of the liquid slug. An illustration of the slug cycle is given in Figure 3. In pipeline-risers systems in the offshore oil industry, these slugs can grow very large, and cause severe problems when they are delivered to the downstream production facility. The inlet separator on the platform will experience large level variation, resulting in poor separation and in some cases flooding. Load variations on the compressors may lead to unnecessary flaring. Another aspect is that the pressure variation caused by slug flow might lead to reduced well performance.

Stabilizing the flow using active control has a great economic potential both in improved regularity and in the possibility for increased recovery of oil. For earlier work on stabilizing slug flow, please consult Hedne and Linga (1990), Henriot et al. (1999) and Havre et al. (2000).

\subsection{Dynamic model of gravity induced slug flow}

We have developed a simplified nonlinear model with 3 states that describes the process (Storkaas et al., 2003). The simplified model is more suitable for control analysis than the conventional PDEbased models used to describe these systems, as it is continuous, low-dimensional, and relatively easy to tune. Storkaas et al. (2003) shows that the model approximates the physical behavior of these systems with sufficient accuracy to be used for controller design and analysis. 
The only actuator for theses systems are usually the downstream production choke. Possible measurement alternatives are upstream pressure $\left(P_{1}\right)$, downstream pressure $\left(P_{2}\right)$ and density $\left(\rho_{T}\right)$ measured just upstream the actuator and volumetric (Q) and mass flow (W) through the production choke $(u=$ Valve opening). The major disturbances is the feed flow, the feed liquid fraction and the downstream separator pressure. We have added first order dynamics to the actuator.

\subsection{Stability analysis}

The bifurcation diagram for the process is given in Figure 4, with valve opening on the horizontal axis and upstream pressure $P_{1}$ at the vertical axis. The solid lines represent open loop stable operation, while the dashed lines indicate unstable operation. Two solid lines for a given valve opening represent a limit cycle were the maximum and minimum pressures are given.

As seen from Figure 4, the process is stable when operated with low choke openings. When the choke opening is increased above $u=0.13$, the process goes through a Hopf bifurcation, resulting in an open loop stable limit cycle (slug flow). However, as seen from the dashed line in Figure 4 there exists unstable, stationary operating points (with a pair of complex RHP poles) for valve openings greater than $u=0.13$. The control problem is thus to design a control system that stabilizes this mode of operation.

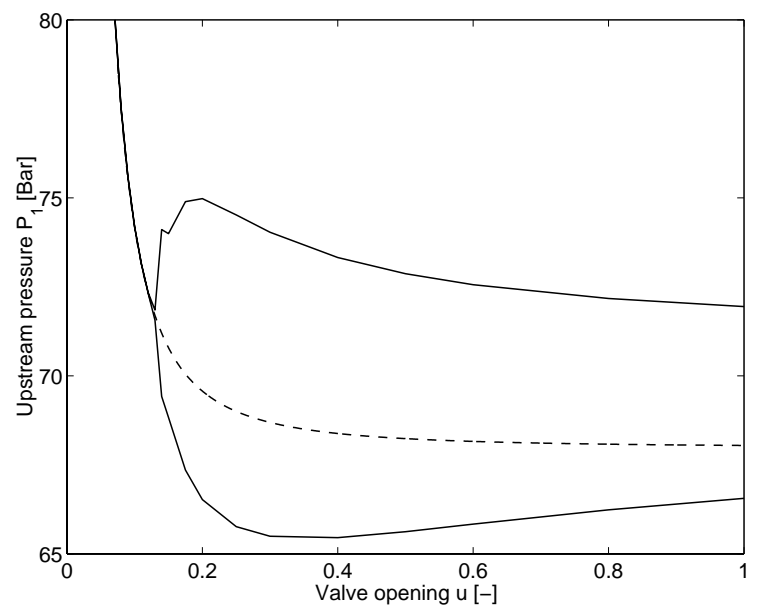

Fig. 4. Bifurcation diagram for the pipeline-riser system

\subsection{Controllability analysis}

The following analysis is performed on a linearized model obtained around a typical unstable operating point (along the dashed line in Figure 4). The same analysis performed at other operating

\begin{tabular}{ccccc}
\hline$P_{1}$ & $P_{2}$ & $\rho_{T}$ & $Q$ & $W$ \\
\hline-0.0034 & 0.0142 & -0.0004 & -4.1173 & -7.6323 \\
- & 3.2489 & 0.0048 & -0.0042 & -0.0004 \\
- & - & - & -0.0004 & 0
\end{tabular}

Table 1. Zeros for different measurement alternatives. Positive (RHP) zeros imply control problems

point show that the results obtained below are typical for this system. It should also be noted that only operating points with valve openings in the low-to-medium range is relevant for closed loop operation. The reason for this is that the system typically is designed with a valve that does not restrict the flow when it is fully opened. A consequence of this is that the pressure drop over the valve at high openings is low, resulting in insufficient process gain for stabilization in this region.

5.3.1. Measurement selection for stabilizing control $\left(y_{2}\right)$ We consider an unstable operating point corresponding to a valve opening of $\mathrm{u}=0.175$. The poles of the system at this operating point is -6.11 and $0.0008 \pm 0.007 i$. The zeros for the different measurement alternatives in Section 5.1 are given in Table 1. The locations of the different measurement alternatives are illustrated in Figure 5.

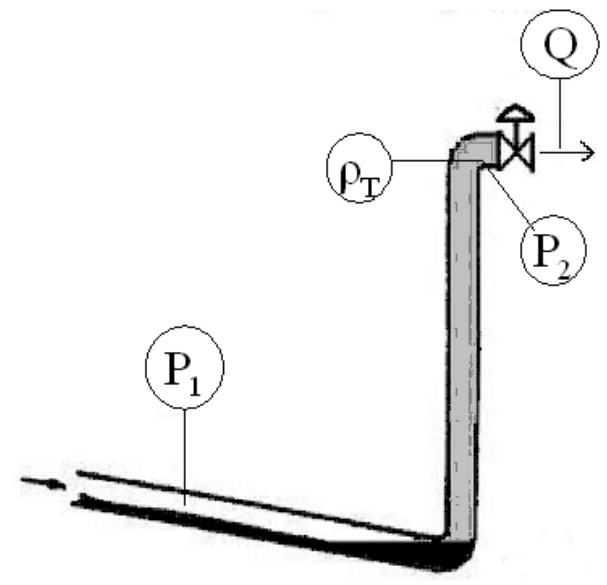

Fig. 5. Measurement locations in pipeline-riser systems

The upstream pressure, $P_{1}$, contains a single left half plane (LHP) zero. This imposes no fundamental control limitations, so $P_{1}$ would thus be a good choice for $y_{2}$. However, this measurement can in many cases be either unreliable or unavailable, and other measurements should also be considered.

Alternatives $P_{2}$ and $\rho_{T}$ in Table 1 both have RHPzeros. With the bandwidth limitation given above (see (3)), we know that we cannot have RHP zeros 
smaller than approximately 0.016 for acceptable performance. Thus, neither $P_{2}$ nor $\rho_{T}$ are suitable as measurements for a stabilizing control due to the bandwidth limitations imposed.

Volumetric flow $Q$ or mass flow $W$ are better alternatives, but both have LHP zeros close to or at the imaginary axis. This means that the steady state gain from $\mathrm{u}$ to $\mathrm{Q}$ or $\mathrm{W}$ is close to or identically zero. Physically, the outflow must equal the inflow at steady state, and the outflow $(Q$ or $W$ ) cannot be set independently. By closing the loop from $\mathrm{u}$ to $Q$ or $W$ (i.e. with a $\mathrm{P}$ controller), we may be able to stabilize the system, but we cannot affect its steady-state behavior, and the system will "drift". This drift may be avoided by measuring another (primary) variable and using a cascade configuration as discussed next.

We choose to control the volumetric flow $\mathrm{Q}\left(y_{2}=\right.$ $Q)$ in the inner loop. We close this loop with an PI controller $K_{2}$ with gain $+8 b a r^{-1}$ and integral time $40 \mathrm{~s}$ (chosen to match the time constant of the valve). The integral action is added under the assumtion that a cascade system is used. With reference to Figure 1 and (4), (5), this results in the following transfer functions:

$$
\begin{array}{r}
G_{2}=\frac{0.00247(s+4.117)(s+0.0042)(s+0.0004)}{(s+0.025)(s+6.112)\left(s^{2}-0.0016 s+0.00005\right)}(6) \\
S_{2}=\frac{s\left(s^{2}-0.0016 s+0.00005\right)}{(s+0.0002)\left(s^{2}+0.011 s+0.0001\right)}(7) \\
G=G_{1} S_{2} K_{2}=G_{1} \frac{8(s+0.025)\left(s^{2}-0.0016 s+0.00005\right)}{(s+0.0002)\left(s^{2}+0.011 s+0.0001\right)}(8)
\end{array}
$$

5.3.2. Choice of primary control variable $y_{1}$ As mentioned, we here assume that we have closed the inner flow control loop $\left(y_{2}=Q\right)$. The above transfer functions show that the complex RHP poles in $G_{2}$ manifest themselves as RHP zeros in $S_{2}$. The choice of measurement in the outer loop $\left(y_{1}\right)$ will determine if the RHP-zeros will appear in $G=G_{1} S_{2} K_{2}$ and thus be a problem for control in the outer loop. Disregarding the choice $y_{1}=P_{1}$ (not measured), we see from Table 1 that $y_{1}=P_{2}$ is probably the best alternative. For this choice

$$
G_{1}=\frac{-0.00007(s-3.249)(s-0.0142)}{(s+0.025)(s+6.112)\left(s^{2}-0.002 s+0.00005\right)}(9)
$$

and the unstable poles in $G_{1}$ will cancel the unstable zeros in $S_{2}$, and $G$ will not contain any RHP zeros due to the unstable poles in $G_{2}$. However, the RHP zero at $z=0.0136$ in $G_{1}$ itself remains. With the bandwidth limitations caused by a real RHP zero given in (1), the closed loop cascade system has an approximate bandwidth limitation of $\omega_{B} \approx 0.0068$ for the outer loop.

One could also choose to use the choke valve position $\left(y_{1}=u\right)$ as a measurement. In this case,
$G_{1}=1$, and the RHP zeros in $S_{2}$ appear in $G$. Now the allowed bandwidth in the outer loop will depend on the frequency of the instability. For the current operating point, the unstable poles of $G_{2}$ is $p=0.0008 \pm 0.007 i$. From (2), the approximate bandwidth limitation for this operating point is $\omega_{B} \approx 0.007$. A change in operating point would result in a change in the bandwidth limitations. For example, for the operating point with a set point in the outer loop of $u=0.25, G_{2}$ has RHP poles on $0.0028 \pm 0.009 i$ resulting in a bandwidth limitation $\omega_{B} \approx 0.0094$.

Comparing $y_{1}=P_{2}$ and $y_{1}=u$ we really have a choice among "two evils"', as the bandwidth will be limited in either cases.

\subsection{Simulation of cascade control of pipeline-riser system}

The above controllability analysis is confirmed by nonlinear simulations on the simplified model. We use a cascade control system with $y_{2}=Q$ in the inner stabilizing loop and $y_{1}=P_{2}$ or $y_{1}=u$ in the outer loop.

The simulations in Figure 6 and 7 are started up in open loop, as can be seen from the initial oscillatory behavior. The controllers are turned on at $t=0.5 \mathrm{hrs}$, with a set point corresponding to the operating point with $u=0.175$ as stationary value. The dashed lines represent the set points. At $t=2.5 \mathrm{hrs}$, the set point is changed to that corresponding to a valve opening of $u=0.25$.
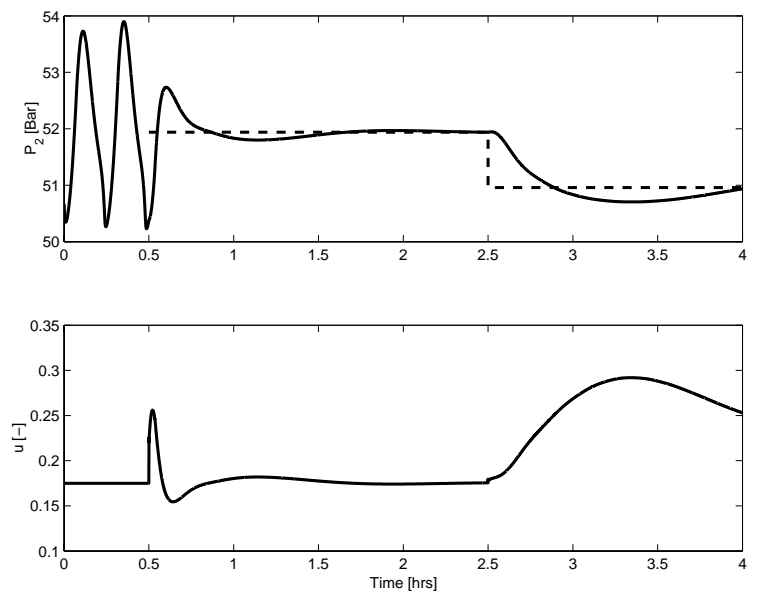

Fig. 6. Stabilizing control with $y_{1}=P_{2}, y_{2}=Q$

$y_{1}=P_{2}$ is the controlled output in Fig. 6. The controller manages to stabilize the process at both operating points, but the response is rather sluggish, especially for the last operating point. This is due to the bandwidth limitations imposed by the RHP zero in $G_{1}$.

In Fig. 7, the valve position is the controlled output $\left(y_{1}=u\right)$. The speed of the response for 

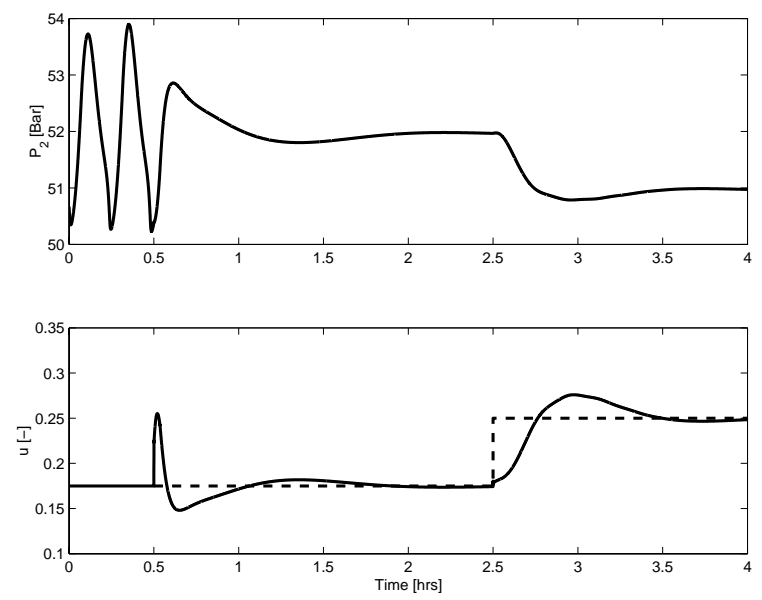

Fig. 7. Stabilizing control with $y_{1}=u, y_{2}=Q$

the first operating point is about the same as for the other controller, where we showed above that the bandwidth limitations were about the same. The response is faster for the second operating point, where the RHP poles in $G_{2}$ are faster.

\subsection{Discussion}

The bandwidth limitations for the outer loop in cascade control structure used above depends on the choice of $y_{1}$. For $y_{1}=P_{2}$, the limitations arise due to the process transfer function in the outer loop, while for $y_{1}=u$, the reason is a RHP zero from the stabilized unstable pole in $G_{2}$. For the studied operating point, the upper bandwidth limit for the two cases are similar. The location of the RHP zeros will be dependent on the operating point, but the dependency is much stronger in the case with $y_{1}=u$. The unstable pole in $G_{1}$ that gives rise to the bandwidth limitation in this case is a strong function of operating point, whereas the RHP zero in $G_{1}$ with $y_{1}=P_{2}$ is almost constant. The controllers must be tuned to deal with the worst-case bandwidth limitations, but the dependency of the RHP zeros to the operating point is nevertheless interesting.

In tuning the controllers, our aim has been to minimize the peaks on the closed loop sensitivity functions $\mathrm{S}$ and $\mathrm{T}$. The reason for choosing this design target is that we want to maximize robustness. We assume an initial state on the limit cycle for the process, which might be far away from the desired operating point. Hence, it is important to be able to bring the process from a wide range of initial conditions into the unstable operating point. For that reason we try to maximize robustness. We have achieved values for $M_{S}$ and $M_{T}$ in the range 1.3-1.6 for the outer loop.

It should also be mentioned that none of the above mentioned RHP zeros are pinned to a certain output, so a multivariable (1 input, multiple outputs) controller (i.e. LQG) would not experience the same bandwidth limitations as the cascade control system discussed above.

\section{CONCLUSIONS}

We have shown that the sensitivity function through a stabilized system will have RHP zeros resulting in inverse response. The RHP zeros may also results in bandwidth limitations for higher level control loops, unless the RHP zeros are cancelled by the same instability in the process through the higher level loops. Slow RHP poles close to the imaginary axis are easy to stabilize but the resulting RHP zeros will be just as slow. In some cases it may lead to improved performance for the control system if one chooses to operate in a more unstable operating point.

The application to stabilization of severe slugging shows that the controllability analysis gives important information for measurement selection and performance limitations.

\section{REFERENCES}

Havre, K., K.O. Stornes and H. Stray (2000). Taming slug flow in pipelines. $A B B$ review 4, 55-63.

Hedne, P. and H. Linga (1990). Supression of terrein slugging with automatic and manual riser choking. Advances in Gas-Liquid Flows pp. 453-469.

Henriot, V., A. Courbot, E. Heintze and L. Moyeux (1999). Simulation of process to control severe slugging: Application to the dunbar pipeline. SPE Annual Conferance and Exibiton in Huston, Texas. SPE 56461.

Holt, B.R. and M. Moriari (1985). Design of resilient processing plants vi - the effect of right plane zeros on dynamic resilience. Chemical Engineering Science 40, 59-75.

Rosenbrock, H.H. (1970). State Space and Multivariable Theory. Nelson, London.

Skogestad, S. and I. Postlethwaite (1996). Multivariable feedback control. John Wiley \& sons.

Skogestad, S., K. Havre and T. Larsson (2002). Control limitations for unstable plants. In: Proc. of IFAC World Congress, Barcelona, 21-26 July 2002, Paper T-Fr-M-15-1.

Storkaas, E., S. Skogestad and J.M. Godhavn (2003). A low-dimentional model of severe slugging for controller design and analysis. In: Proc. of MultiPhase '03, San Remo, Italy, 1113 June 2003. 